\title{
Emulsified isoflurane combined with therapeutic hypothermia improves survival and neurological outcomes in a rat model of cardiac arrest
}

\author{
MENG-JUN WU*, YA-JIE ZHANG*, HAI YU and BIN LIU \\ Department of Anesthesiology, West China Hospital, Sichuan University, Chengdu, Sichuan 610041, P.R. China
}

Received August 18, 2016; Accepted November 28, 2016

DOI: $10.3892 / \mathrm{etm} .2017 .4044$

\begin{abstract}
Emulsified isoflurane (EIso), when introduced following cardiopulmonary resuscitation (CPR), significantly improves survival and neurological outcomes in a rat model of cardiac arrest $(\mathrm{CA})$. The present study aimed to examine whether EIso combined with therapeutic hypothermia (TH) confers an additive neuroprotective effect. Adult male Sprague-Dawley rats that were subjected to return of spontaneous circulation (ROSC) after a 6-min asphyxia-induced CA were randomized to five groups: Sham group, control group under normothermic conditions, EIso group ( $4 \mathrm{ml} / \mathrm{kg}$ for $30 \mathrm{~min}$ under normothermic conditions), $\mathrm{TH}$ group $\left(33^{\circ} \mathrm{C}\right.$ for $2 \mathrm{~h}$ ), and EIso plus TH group. Survival conditions and neurological outcomes were evaluated at 1 day and 7 days after ROSC. Animal survival rate at 7 days after ROSC was $30.7 \%$ in the CPR group, $60 \%$ in the EIso group, $63.6 \%$ in the $\mathrm{TH}$ group and $72.7 \%$ in the EIso plus $\mathrm{TH}$ group. EIso, TH and EIso plus TH yielded significant improvements in survival rates, neural deficit score and cognitive function, and ameliorated hippocampal CA1 region cell injury and apoptosis at 1 day and 7 days after ROSC compared with the CPR group. Combined therapy of EIso and TH was superior to EIso or TH alone, suggesting that combined EIso and $\mathrm{TH}$ treatment results in significant improvements in survival and neurological outcomes, and was more effective than independent EIso or TH treatment.
\end{abstract}

\section{Introduction}

In past two decades, the survival and discharge rates following cardiac arrest $(\mathrm{CA})$ with neurological sequelae remain a clinical problem; for instance, the survival rate following

Correspondence to: Dr Hai Yu or Dr Bin Liu, Department of Anesthesiology, West China Hospital, Sichuan University, 37 Guoxue Alley, Chengdu, Sichuan 610041, P.R. China

E-mail: yuhaishan117@yahoo.com

E-mail: 2014982567@qq.com

*Contributed equally

Key words: emulsified isoflurane, therapeutic hypothermia, brain, cardiopulmonary resuscitation, cardiac arrest hospital admission was reported to be $25 \%$, and was $<15 \%$ in the 5 years following resuscitation $(1,2)$.

Mild therapeutic hypothermia (TH) is an effective treatment approach for organ protection following CA $(3,4)$. However, this does not provide complete neuroprotection (5) and is still underutilized in CA treatment (6), which necessitates investigation into additional agents to improve clinical outcomes when combined with $\mathrm{TH}$. Increasing evidence suggests that $\mathrm{TH}$ combined with other treatments may represent a helpful strategy to provide better protection for the brain and other organs subsequent to the return of spontaneous circulation (ROSC) (5,7-13). Fries et al (12) reported that during induction of mild TH, even a short exposure to xenon provided significant improvements in functional recovery and ameliorated myocardial dysfunction in a pig model of CA. Meybohm et al (13) reported that a combination of $\mathrm{TH}$ and sevoflurane postconditioning reduced myocardial and cerebral damages following cardiopulmonary resuscitation (CPR). Therefore, combined therapy of $\mathrm{TH}$ and other treatments appears to be a more effective approach.

Emulsified isoflurane (EIso), the emulsified preparation of isoflurane, in a recent study by the present authors, was revealed to have neuroprotective effects on CA (14). In this previous study, EIso ( $4 \mathrm{ml} / \mathrm{kg}$ ) improved survival rate, neural deficit score (NDS) and memory function, and ameliorated hippocampal CA1 region cell death and apoptosis at 7 days after ROSC. Furthermore, a phase I clinical trial of Eiso was recently performed in West China Hospital (Sichuan University, Chengdu, China), and the results demonstrated that EIso had marked anesthetic potency and may be used safely in patients (15). In addition, EIso has many potential therapeutic applications for multiple-organ protection in the heart (16-19), kidneys (20), liver and lungs (21) when induced by ischemia/reperfusion (I/R) injury. Therefore, it was hypothesized in our previous study (14) that EIso combined with TH may produce additive efficacy compared with either treatment alone following CPR. The present study was designed to evaluate whether a combination of EIso and TH achieves better survival and neurological outcomes compared with either treatment alone.

\section{Materials and methods}

Animal and drug preparations. The present study was approved by the Institutional Animal Care and Use Committee of Sichuan University. All animals were maintained in 
compliance with the Guide for the Care and Use of Laboratory Animals. A total of 65 adult male Sprague-Dawley rats (age, 3 months) weighing 250-350 g were used (West China Animal Breeding Centre of Sichuan University, China). These were maintained at a room temperature of $27 \pm 2^{\circ} \mathrm{C}$, with a $12 \mathrm{~h}$ light-dark cycle. In accordance with a previous protocol (15), EIso was prepared in our laboratory. Briefly, $18.4 \mathrm{ml} 30 \%$ intralipid (Sino-Swed Pharmaceutical Corp. Ltd., Beijing, China) and $1.6 \mathrm{ml}$ liquid isoflurane (Abbott Pharmaceutical Co., Ltd., Lake Bluff, IL, USA) were mixed in a 20-ml glass ampoule, which was sealed using an alcohol blowtorch. The concentration of EIso (8\%) was rechecked by gas chromatography (4890 D; Agilent Technologies, Inc., Santa Clara, CA, USA) at the beginning of experiments.

CA model. All animals were fasted overnight, but had free access to water. All procedures were performed under sterilized conditions. Rats were anesthetized with $10 \%$ chloral hydrate (Sigma-Aldrich; Merck KGaA; $30 \mathrm{mg} / 100 \mathrm{~g}$ ) intraperitoneally, intubated with a 16-gauge cannula (B. Braun Melsungen AG, Melsungen, Germany) and mechanically ventilated with a tidal volume of $10 \mathrm{ml} / \mathrm{kg}$, a respiratory rate of 60 breaths/min, and a fraction of inspired oxygen $\left(\mathrm{FiO}_{2}\right)$ of 0.21 for $10 \mathrm{~min}$. The left femoral artery and vein were cannulated with 24-gauge and 22-gauge catheters, respectively. The mean arterial pressure (MAP) and heart rate were monitored continuously by a BL-420E biological and functional experiment recorder (Chengdu Technology Co., Ltd., Chengdu, China. The rat core temperature was maintained at $37 \pm 0.5^{\circ} \mathrm{C}$ with a rectal temperature probe. Vecuronium (Yichang Humanwell Pharmaceutical Co. Ltd., Yichang, China) $(1 \mathrm{mg} / \mathrm{kg} / \mathrm{h}$, intravenously) was used for immobilization and was administered prior to asphyxia. After $6.0 \mathrm{~min}$ of asphyxiation (approximately $3.5 \mathrm{~min}$ of CA) without ventilation, which was achieved by clamping the tracheal tube, mechanical ventilation was restarted with $100 \%$ oxygen, at a rate of 80 breaths/min and a tidal volume of $10 \mathrm{ml} / \mathrm{kg}$. Epinephrine $(0.02 \mathrm{mg} / \mathrm{kg}$; Yichang Humanwell Pharmaceutical Co., Ltd.) and sodium bicarbonate ( $1 \mathrm{mg} / \mathrm{kg}$; Yichang Humanwell Pharmaceutical Co., Ltd.) were administered intravenously, followed by rapid manual chest compressions until ROSC was achieved.

Experimental protocol. A total of 5 min after achieving ROSC (Fig. 1), rats were randomly assigned to five groups, as follows: i) Sham group (sham-operated group; animals were not subjected to ischemia/reperfusion, and no additional medication was administered except Ringer's lactate solution); ii) $\mathrm{CPR}$, under normothermia $\left(37^{\circ} \mathrm{C}\right)$; iii) $\mathrm{TH}\left(33^{\circ} \mathrm{C}\right.$ for $\left.2 \mathrm{~h}\right)$; iv) EIso (intravenous $4 \mathrm{ml} / \mathrm{kg}$ EIso for $30 \mathrm{~min}$ under normothermia); and v) EIso plus TH groups (intravenous $4 \mathrm{ml} / \mathrm{kg}$ EIso for 30 min under $33^{\circ} \mathrm{C}$ for $2 \mathrm{~h}$ ).

In animals assigned to the $\mathrm{TH}$ group, body cooling was initiated 5 min after ROSC. Rectal temperature was reduced to $33^{\circ} \mathrm{C}$ within $10 \mathrm{~min}$ with the aid of ice packs and fans. Once reached, the target temperature was maintained for $2 \mathrm{~h}$ and then returned to $37^{\circ} \mathrm{C}$ at a rate at $1^{\circ} \mathrm{C} / \mathrm{h}$, similarly to a previous study (22). Following a recovery period, rats were weaned from the ventilator, all vascular catheters and tracheal tubes were removed and surgical wounds were sutured. Subsequent to extubation, rats were observed in $\mathrm{FiO}_{2}$ of 1.0 for $30 \mathrm{~min}$, then returned to a chamber to breathe normal air for $30 \mathrm{~min}$. A cardiac rhythm with a MAP of $>60 \mathrm{mmHg}$ for a minimum of 5 min was defined as successful ROSC. CPR was continued unless the animal was either successfully resuscitated or pronounced dead after a total of $15 \mathrm{~min}$ CPR.

The survival rate study used 65 rats; 9 of these were excluded from subsequent analyses (4 rats did not achieve successful ROSC; CA was not induced due to a technical failure in 4 rats; and an excessive EIso dose was administered in 1 rat). Randomization occurred following ROSC. The 7-day survival rate and a survival curve were evaluated for all five groups. All evaluations were processed at 1 day prior to the operation (baseline), and at 1 and 7 days after ROSC by one investigator, who was blinded to the experimental groups.

NDS. NDS was determined using a scale from a previous study (23). The neurological function system consists of five components: Baseline metrics (body weight, heart rate, body temperature), cranial nerve function, motor function, sensory function, and coordination. A normal rat has an NDS of 500, and a score of 0 is attained at mortality.

Cognitive function. The fear potentiated startle reflex test was used to evaluate rats' cognitive function at 1 day before CPR (baseline), 1 day and 7 days (all measured in the same rats) after ROSC, in accordance with a previous study (24). After a 5-min acclimation period, noise bursts (startle stimuli) were applied at either 100 or $110 \mathrm{~dB}$, lasting $50 \mathrm{msec}$ each. These were imposed either alone or after $2.5 \mathrm{sec}$ of light emittance, with the light continuing to $3 \mathrm{sec}$. This provided a total of 9 trials. The extent to which each rat jumped in response to the noise in the presence and absence of light was measured by the average acceleration generated by the jump over the $100 \mathrm{msec}$ after initiation of the noise; cognitive function was considered to be decreased when the average acceleration of the startle was less than control group. Following this test, rats were trained for potentiated startle. After 5-min acclimation period, rats received $0.7 \mathrm{~mA}$ shocks to their feet, delivered during the last $0.5 \mathrm{sec}$ of a 3 -sec light presentation. The average light interval duplicated that of the above protocol. Following this training, rats were tested for potentiated startle. No shocks were given during testing. After a 5-min acclimation period, rats received a startle stimulus (noise burst) alone or in conjunction with a light presentation as in the matching protocol. A greater response to noise plus light indicated potentiating, i.e., that the rat had learned the association between the light and a shock.

Histopathological analysis. At 7 days after ROSC, rats were perfused with phosphate-buffered saline under anesthesia with $10 \%$ chloral hydrate, followed by $4 \%$ paraformaldehyde. Subsequent to perfusion, rats were decapitated; brains were removed carefully and postfixed in formalin for 24-48 h. Following this, dehydration was performed in graded concentrations of ethanol and butanol and paraffin-embedded coronal sections (10- $\mu \mathrm{m}$ thick) were made at the hippocampal level (approximately at the bregma, $-3.0 \mathrm{~mm}$ ). For the detection of DNA fragmentation, terminal deoxynucleotidyl transferase-mediated dUTP nick end labeling (TUNEL) staining was used as previously described (25). At a magnification of 400x, the hippocampal CA-1 sector was analyzed 
Table I. Physiological variables at the baseline, 30,60 and $120 \mathrm{~min}$ after the return of spontaneous circulation.

\begin{tabular}{|c|c|c|c|c|c|c|c|c|}
\hline Group & $\begin{array}{l}\text { Time, } \\
\text { mins }\end{array}$ & $\begin{array}{l}\text { MAP, } \\
\mathrm{mmHg}\end{array}$ & $\begin{array}{l}\text { HR, } \\
\text { bpm }\end{array}$ & $\mathrm{pH}$ & $\begin{array}{c}\mathrm{PaCO}_{2} \\
\mathrm{mmHg}\end{array}$ & $\begin{array}{l}\mathrm{LAC}, \\
\mathrm{mmol} / \mathrm{l}\end{array}$ & $\begin{array}{c}\mathrm{BE}, \\
\mathrm{mmol} / \mathrm{l}\end{array}$ & $\begin{array}{l}\mathrm{HCO}_{3^{-}} \\
\mathrm{mmol} / \mathrm{l}\end{array}$ \\
\hline \multirow[t]{4}{*}{ CPR } & Baseline & $95.6 \pm 19.6$ & $367 \pm 59$ & $7.34 \pm 0.49$ & $35 \pm 8$ & $1.1 \pm 0.5$ & $-6.9 \pm 2.7$ & $18.9 \pm 2.0$ \\
\hline & 30 & $72.9 \pm 14.7$ & $351 \pm 46$ & $7.29 \pm 0.07$ & $41 \pm 12$ & $3.1 \pm 0.7$ & $-10.4 \pm 2.4$ & $16.0 \pm 2.3$ \\
\hline & 60 & $73.2 \pm 16.5$ & $365 \pm 31$ & $7.28 \pm 0.06$ & $46 \pm 7$ & $2.5 \pm 0.3$ & $-6.0 \pm 2.5$ & $19.1 \pm 2.2$ \\
\hline & 120 & $72.3 \pm 17.2$ & $351 \pm 48$ & $7.32 \pm 0.07$ & $42 \pm 13$ & $2.4 \pm 0.6$ & $-5.8 \pm 2.7$ & $18.8 \pm 3.5$ \\
\hline \multirow[t]{4}{*}{ EIso } & Baseline & $97.9 \pm 17.8$ & $362 \pm 72$ & $7.33 \pm 0.03$ & $34 \pm 3$ & $1.7 \pm 0.6$ & $-7.5 \pm 2.6$ & $18.6 \pm 1.7$ \\
\hline & 30 & $71.3 \pm 14.4$ & $359 \pm 43$ & $7.24 \pm 0.08$ & $35 \pm 10$ & $3.7 \pm 1.4$ & $-11.5 \pm 3.6$ & $15.3 \pm 2.3$ \\
\hline & 60 & $75.4 \pm 16.9$ & $364 \pm 49$ & $7.23 \pm 0.09$ & $47 \pm 13$ & $2.5 \pm 1.0$ & $-7.6 \pm 2.5$ & $17.6 \pm 2.0$ \\
\hline & 120 & $73.8 \pm 13.8$ & $351 \pm 39$ & $7.32 \pm 0.12$ & $46 \pm 11$ & $2.3 \pm 0.4$ & $-5.6 \pm 3.4$ & $17.8 \pm 3.1$ \\
\hline \multirow[t]{4}{*}{ EIso+TH } & Baseline & $101.6 \pm 14.2$ & $341 \pm 56$ & $7.35 \pm 0.04$ & $34 \pm 6$ & $1.0 \pm 0.3$ & $-6.1 \pm 2.2$ & $19.6 \pm 1.9$ \\
\hline & 30 & $72.6 \pm 13.6$ & $323 \pm 30$ & $7.33 \pm 0.09$ & $43 \pm 17$ & $3.2 \pm 1.5$ & $-9.7 \pm 2.3$ & $16.0 \pm 1.7$ \\
\hline & 60 & $78.3 \pm 14.3$ & $366 \pm 42$ & $7.25 \pm 0.06$ & $47 \pm 8$ & $2.6 \pm 0.4$ & $-6.1 \pm 2.7$ & $18.4 \pm 1.9$ \\
\hline & 120 & $74.2 \pm 12.7$ & $361 \pm 32$ & $7.28 \pm 0.11$ & $44 \pm 12$ & $2.3 \pm 0.4$ & $-5.7 \pm 2.5$ & $18.0 \pm 2.7$ \\
\hline \multirow[t]{4}{*}{ TH } & Baseline & $104.9 \pm 15.6$ & $371 \pm 58$ & $7.31 \pm 0.08$ & $32 \pm 4$ & $1.2 \pm 0.3$ & $-6.2 \pm 3.8$ & $19.1 \pm 3.0$ \\
\hline & 30 & $74.5 \pm 13.9$ & $342 \pm 35$ & $7.27 \pm 0.09$ & $36 \pm 12$ & $4.1 \pm 1.5$ & $-12.5 \pm 3.5$ & $15.2 \pm 2.4$ \\
\hline & 60 & $75.6 \pm 11.2$ & $359 \pm 33$ & $7.28 \pm 0.06$ & $48 \pm 16$ & $2.5 \pm 0.2$ & $-7.5 \pm 2.1$ & $17.7 \pm 1.8$ \\
\hline & 120 & $75.2 \pm 13.5$ & $341 \pm 43$ & $7.26 \pm 0.10$ & $45 \pm 12$ & $2.3 \pm 0.6$ & $-5.5 \pm 3.6$ & $17.3 \pm 4.5$ \\
\hline
\end{tabular}

All values are reported as mean \pm standard deviation. No differences were observed between the groups. CPR, cardiopulmonary resuscitation; EIso, emulsified isoflurane; TH, therapeutic hypothermia; MAP, mean arterial pressure; HR, heart rate; $\mathrm{PaCO}_{2}$, partial pressure of $\mathrm{CO}_{2}$; $\mathrm{LAC}_{\text {, }}$ lupus anti-coagulant; BE, base excess; bpm, beats per minute.

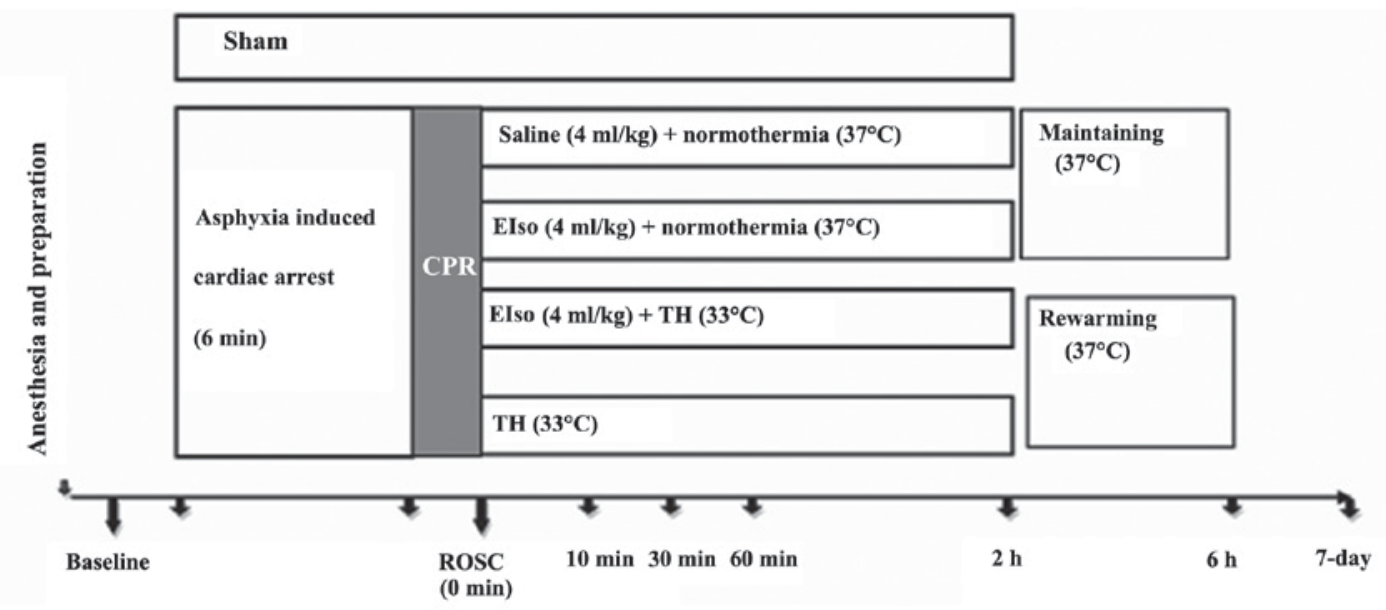

Figure 1. Experimental protocol for CPR and postresuscitation care in an asphyxia-induced cardiac arrest model. ROSC, return of spontaneous circulation; $\mathrm{CPR}$, cardiopulmonary resuscitation; EIso, emulsified isoflurane; TH, therapeutic hypothermia.

thoroughly by counting all TUNEL-positive cells (Vivien v. 1.2.2; CAST, Toronto, ON, Canada). For evaluations of the proportion of viable neurons in the hippocampal CA-1 sector, brain sections were stained with toluidine blue to mark Nissl bodies at $10-\mu \mathrm{m}$ sections at the same level as the hippocampus. These examinations were made by a pathologist who was blinded to the experimental groups.

Statistical analysis. Data are expressed as the mean \pm standard deviation. Homogeneity of variance was evaluated using Levene's test. Physiological variables, duration of CA and the viable neuron count were analyzed by one-way analysis of variance with least significant difference correction for post hoc comparisons between multiple experimental groups. Survival rate was compared by the Kaplan-Meier method with a log-rank test. Statistical analysis was performed using SPSS software 18.0 (SPSS, Inc., Chicago, IL, USA). P<0.05 was considered to represent a statistically significant difference.

\section{Results}

There were no differences amongst the 5 experimental groups with regard to hemodynamics and arterial blood gases, as reported in Table I. Times taken for the CPR process, including 
CA, asphyxia and CPR time were not significantly different (Table II).

The survival rate at 7 days after ROSC was 4 of 13 rats (30.7\%) in the control group, 6 of 10 rats $(60 \%)$ in the EIso group, 7 of 11 rats $(63.6 \%)$ in the TH group and, 8 of 11 rats $(72.7 \%)$ in the EIso plus TH group ( $\mathrm{P}<0.05$ vs. control group; Fig. 2 ).

The NDS was evaluated at 1 and 7 days after ROSC. NDS values at 1 and 7 days after ROSC were significantly higher in the EIso $(448 \pm 24,469 \pm 34 ; \mathrm{P}=0.014)$, TH $(452 \pm 36$, $482 \pm 32 ; \mathrm{P}=0.035)$, and EIso plus TH groups $(467 \pm 40,492 \pm 15$; $\mathrm{P}=0.021)$ than in the CPR group $(414 \pm 32,445 \pm 40)$ (Fig. 3A), and the scores in the EIso plus TH group were significantly higher than in the TH or EIso groups. Improved neurological outcomes after EIso treatment at 1 and 7 days were consistent with our unpublished work.

Rats were also subjected to a fear potentiated startle reflex test to assess cognitive function at 1 and 7 days after ROSC. The EIso, TH, and EIso plus TH groups had improved cognitive function $(528.2 \pm 316.2, \mathrm{P}=0.004 ; 378.2 \pm 132, \mathrm{P}=0.043$; and $315.4 \pm 134, \mathrm{P}=0.017$, respectively) at 1 day and $(1,834.5 \pm 432.2$, $\mathrm{P}=0.002 ; 1,638.2 \pm 332.4, \mathrm{P}=0.025$; and $1,416.4 \pm 234.7, \mathrm{P}=0.031$, respectively) at 7 days after ROSC, compared with the CPR group $(84.5 \pm 32.2,728.2 \pm 326.3)$, whereas these values in the EIso plus TH group were higher than in the TH or EIso group (Fig. 3B).

A transient duration of global cerebral ischemia/reperfusion during CPR and following CA is associated with delayed neuronal death in selectively vulnerable areas such as the hippocampus (26). The percentage of viable hippocampal CA-1 pyramid neurons (stained Nissl bodies) in the CPR group was significantly less than in response to the three treatment groups at 7 days after $\operatorname{ROSC}(\mathrm{P}=0.016)$, whereas the percentage of normal neurons in EIso plus TH was enhanced compared with the EIso or TH groups (Figs. 4 and 5A). As reported in Figs. 4 and 5B, the number of TUNEL-positive cells in the hippocampal CA-1 region was significantly lower in the EIso, TH, and EIso plus TH groups compared with the CPR group $(\mathrm{P}=0.041$ for EIso and $\mathrm{TH} ; \mathrm{P}=0.003$ for EIso plus $\mathrm{TH}$ vs. control). Furthermore, the number of TUNEL-positive cells was significantly lower in the EIso plus TH group compared with the EIso group $(\mathrm{P}=0.039)$, and TH group $(\mathrm{P}<0.05)$ but this was not significantly different between EIso group and TH group ( $\mathrm{P}>0.05)$. Combined therapy of EIso and TH was therefore concluded to be superior to EIso or TH alone.

\section{Discussion}

In the present study, a combination of EIso and TH treatment had an additive effect in improving both survival and neurological outcomes subsequent to ROSC in a rat model, and was more effective than EIso or TH alone.

Neurological dysfunction resulting from CA contributes to morbidity and mortality following initially successful CPR $(27,28)$. To date, TH is the only intervention confirmed to improve neurological recovery (27). Despite this, the mortality rate of patients admitted to critical care units after CA remain high, and severe neurological sequelae are common among those who survive $(29,30)$. Previous studies have reported that $\mathrm{TH}$ combined with other treatments is a helpful strategy to improve survival rate and neurological outcomes subsequent
Table II. Time taken for CA, asphyxia and ROSC, measured in sec.

\begin{tabular}{lccc}
\hline Group & CA & Asphyxia & ROSC \\
\hline CPR & $243 \pm 46$ & $117 \pm 42$ & $76 \pm 27$ \\
EIso & $238 \pm 40$ & $125 \pm 34$ & $81 \pm 36$ \\
EIso+TH & $225 \pm 35$ & $132 \pm 41$ & $74 \pm 40$ \\
TH & $228 \pm 31$ & $130 \pm 38$ & $75 \pm 24$ \\
\hline
\end{tabular}

All values are reported as mean \pm standard deviation. No differences were observed between the groups. CA was defined as time from stopping ventilation to $\mathrm{CA}$; asphyxia was defined as time from $\mathrm{CA}$ to starting CPR; ROSC was defined as time from starting CPR to ROSC. CPR, cardiopulmonary resuscitation; EIso, emulsified isoflurane; $\mathrm{TH}$, therapeutic hypothermia; $\mathrm{CA}$, cardiac arrest; ROSC, return of spontaneous circulation.

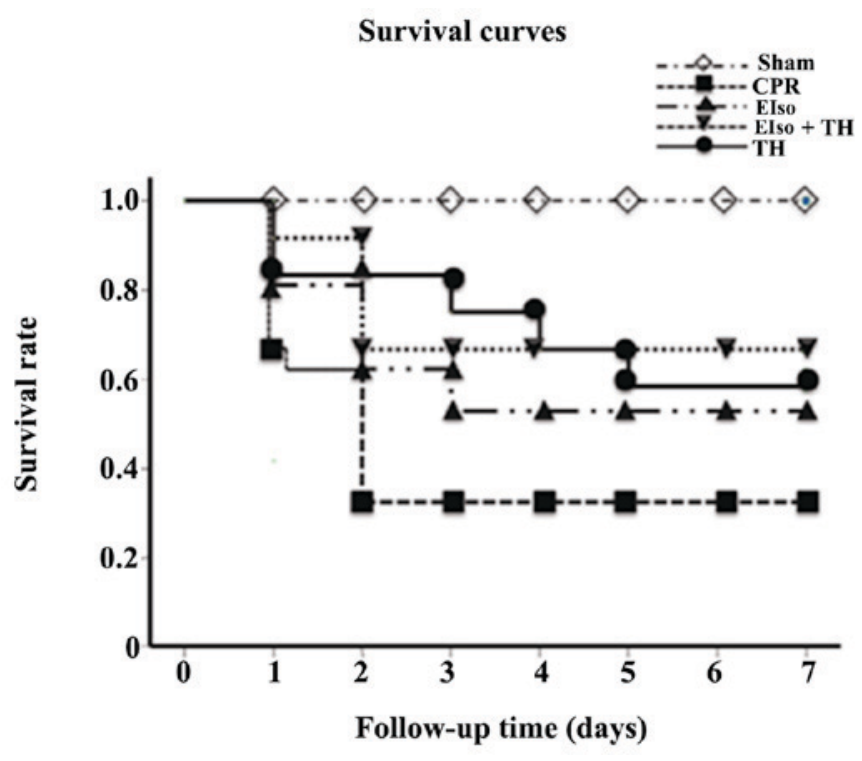

Figure 2. Kaplan-Meier analyses of cumulative survival in the 7 days after return of spontaneous circulation. $\mathrm{P}<0.05$ for EIso, for EIso plus $\mathrm{TH}$ and for TH vs. CPR group. CPR, cardiopulmonary resuscitation; EIso, emulsified isoflurane; $\mathrm{TH}$, therapeutic hypothermia.

to ROSC (27). In the present study, the combination of EIso and $\mathrm{TH}$ similarly demonstrated an additive effect in improving survival and neurological outcomes following ROSC.

The survival rate $(72.7 \%)$ in the EIso plus $\mathrm{TH}$ group at 7 days after ROSC was higher than that of the TH (63.6\%) or EIso $(60 \%)$ groups alone, although this was significantly different. This result was consistent with previous studies using a rat model of 6-minute CA $(11,31)$. Ma et al (31) demonstrated that 4 of 10 animals survived $>72 \mathrm{~h}$ in a cannabinoid receptor agonist WIN55, 212-2 hypothermia group, and Hayashida et al (11) reported $77 \%$ (10/13) survival in an $\mathrm{H}_{2}$ plus $\mathrm{TH}$ group after $72 \mathrm{~h}$. Furthermore, the experimental NDS and average value in the fear potentiated startle reflex test at 1 and 7 days after ROSC in the EIso plus TH group was also higher than that of the TH and EIso groups. As mentioned above, average value is to reflect cognitive capability of rats, and higher average value demonstrates 
A

B
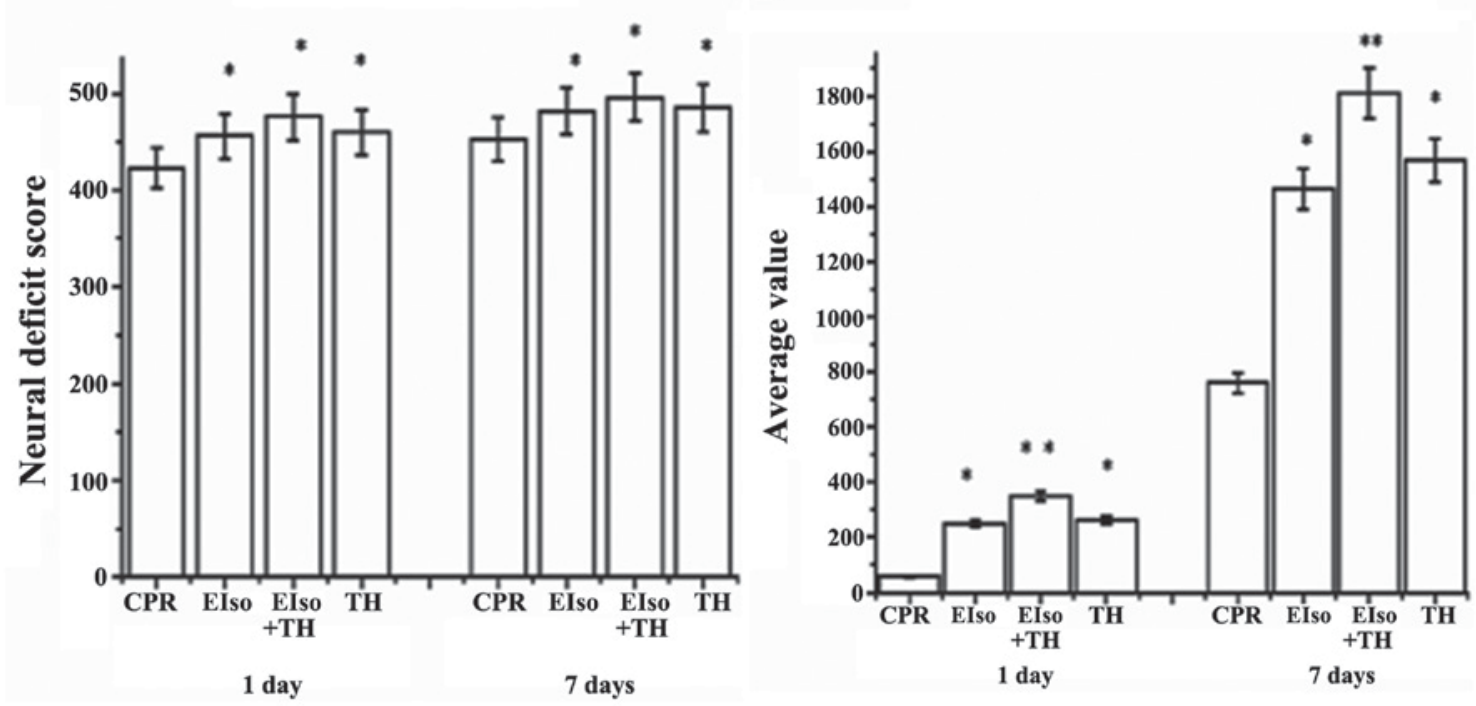

Figure 3. Behavioral evaluation at 1 and 7 days following return of spontaneous circulation. (A) Neural deficit score; (B) Fear potentiated startle reflex test. ${ }^{*} \mathrm{P}<0.05$; and ${ }^{* *} \mathrm{P}<0.005$ vs. CPR group. CPR, cardiopulmonary resuscitation; EIso, emulsified isoflurane; TH, therapeutic hypothermia.

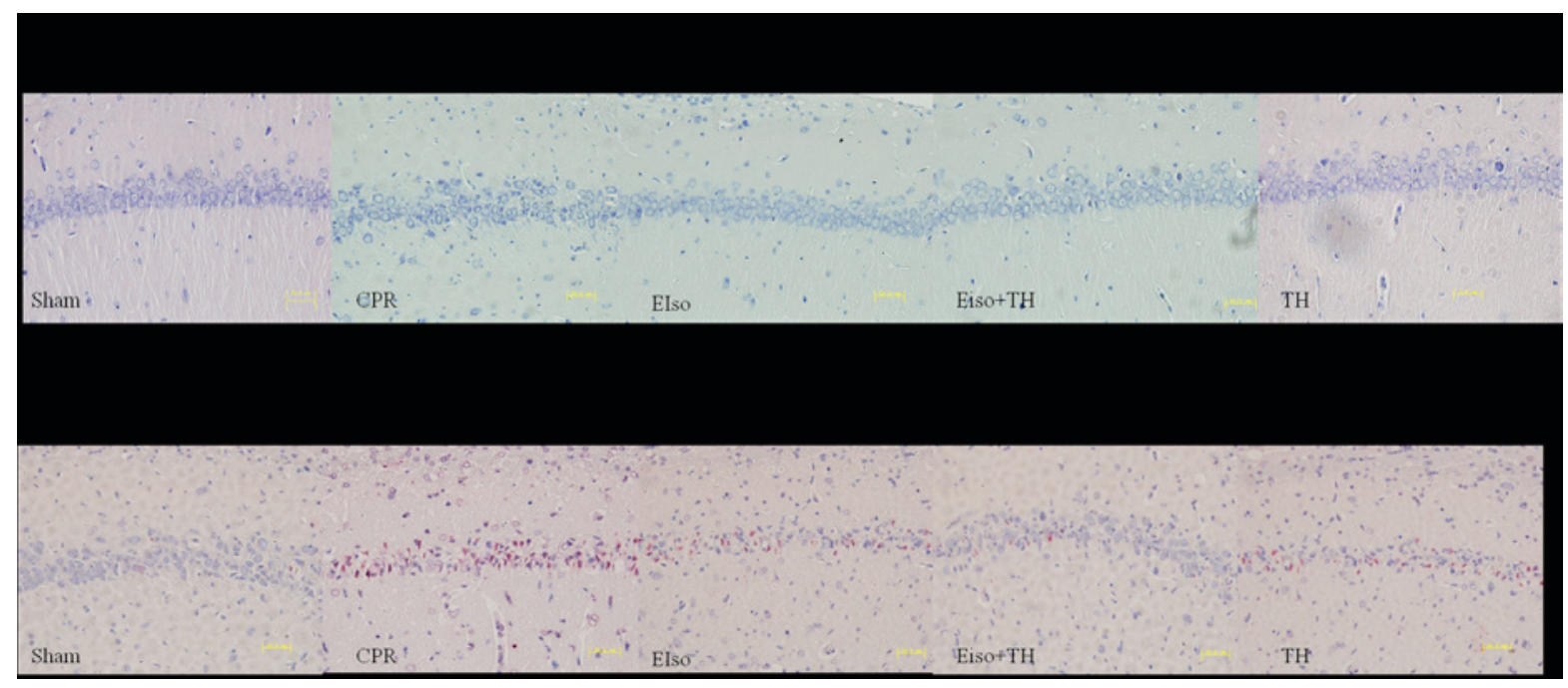

Figure 4. Staining of Nissl bodies with toluidine blue, and TUNEL-stained hippocampal CA-1 zone at 7 days after return of spontaneous circulation. CPR, cardiopulmonary resuscitation; EIso, emulsified isoflurane; TH, therapeutic hypothermia; TUNEL, terminal deoxynucleotidyl transferase dUTP nick end labeling.

better cognitive function. Based on this theory, combined therapy of EIso plus TH was superior to TH or EIso alone in improving neurological function and cognitive function at 1 and 7 days after ROSC.

Neuronal degeneration in the hippocampal CA-1 region was suppressed by both TH alone and EIso alone, and the combined therapy of EIso plus TH was most effective. Brain histopathological analysis revealed that the percentage of viable Nissl neurons at 7 days after ROSC in the EIso plus TH group was significantly preserved in comparison to that in the $\mathrm{TH}$ and EIso groups, and fewer TUNEL-stained apoptotic neurons were reported in the EIso plus TH group than that in the in the TH and EIso groups. Therefore, based on these data, a combination treatment of EIso and TH demonstrates advantages in response to cerebral ischemia/reperfusion injury compared with $\mathrm{TH}$ or EIso treatment alone in a rat model of CA. These results were consistent with previous studies in a rat of $\mathrm{CA}$; at 7 days after ROSC, combined therapy reduced the proportion of apoptotic cells and protected the living neurons compared with other treatments $(5,9-11,31)$. In the current study, it was confirmed that the animals treated with EIso after CPR retained significantly better brain functions than the control group; this demonstrated that the combination of EIso and TH showed an additive effect in improving both survival and neurologic outcomes after ROSC. Combined therapy of TH with other treatments appears the most effective approach. Furthermore, if the protective effect of intravenous EIso combined with $\mathrm{TH}$ is confirmed by additional, larger clinical trials, the combined therapy of EIso and TH may represent a promising approach for induction of mild hypothermia in or following CA.

The survival and neurologic outcomes after ROSC in the EIso plus TH group were superior than in the $\mathrm{TH}$ alone and 
A

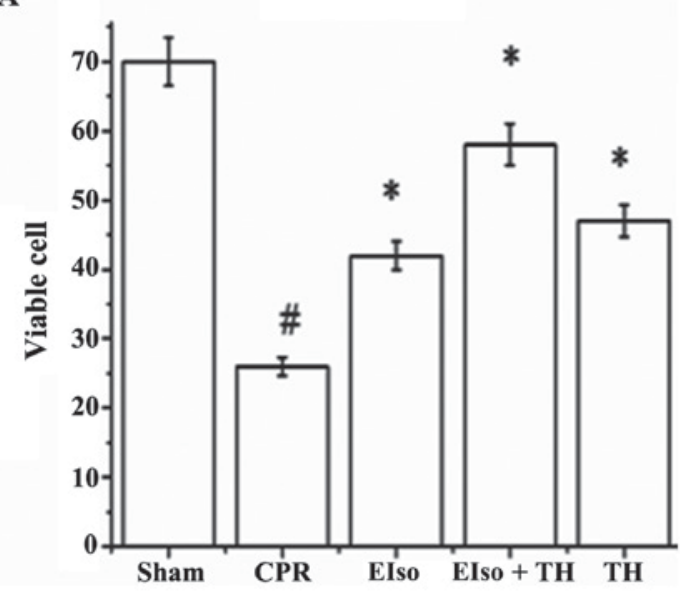

B

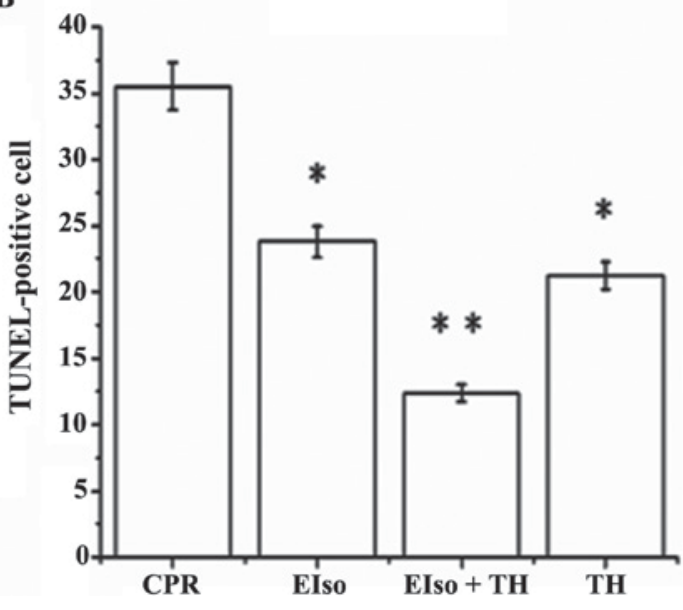

Figure 5. (A) Viable cells in Nissl staining and (B) TUNEL-positive cells in the hippocampus CA1 at 7 days after return of spontaneous circulation. Significant differences: " $\mathrm{P}<0.005$ vs. sham group; ${ }^{*} \mathrm{P}<0.05$; and ${ }^{* *} \mathrm{P}<0.005$ vs. CPR group. CPR, cardiopulmonary resuscitation; EIso, emulsified isoflurane; TH, therapeutic hypothermia; TUNEL, terminal deoxynucleotidyl transferase dUTP nick end labeling.

EIso group alone. The additive effect suggested that both EIso and hypothermia may share common mechanisms of neuroprotection, eliminating reactive oxygen species generation and inhibiting the expression of caspase-3, leading to cellular apoptosis and massive autophagy in rats experiencing ischemia/reperfusion injury (14,32-34).

In the present study and our previous unpublished work, EIso was demonstrated to significantly ameliorate cellular apoptosis and improve neuroprotection. There were a number of reasons for no significant differences among three groups: First, in the current model, EIso $(4 \mathrm{ml} / \mathrm{kg}$ ) was only administered for a short, 0.5 -h period but yielded an effect nonetheless. In our previous unpublished work, although $4 \mathrm{ml} / \mathrm{kg}$ EIso was more effective in terms of survival rate and neurological outcomes than $2 \mathrm{ml} / \mathrm{kg}$ EIso, the dose-effect relationship between EIso and cerebral ischemic protection requires additional exploration. Second, the duration of this 3.5 min CA may be shorter than that experienced by patients. However, it was hypothesized that lower brain neuron injury would be observed under this short duration of CA, and that EIso plus TH may still achieve an addictive effect. A potential limitation is that a longer duration may have significant effects (35), although the brain morphology demonstrated significant differences between treatment and control groups following a 3.5-min CA.

Previous experimental studies have suggested that the benefit afforded by hypothermia is closely linked to the timing of $\mathrm{TH}$, specifically the rapidity in body temperature decrease following resuscitation and rewarming rate (9-11). In present study, TH at a target temperature of $33^{\circ} \mathrm{C}$ within 15 min following CA and a rewarming rate at $1^{\circ} \mathrm{C} / \mathrm{h}$ were typically reached in this rat model $(5,9-11,31)$. Lu et al (22) demonstrated that a rewarming rate at $0.5-1^{\circ} \mathrm{C} / \mathrm{h}$ reduced the severity of myocardial and cerebral injuries, and inflammatory reaction after CPR; a rapid rewarming rate at $2^{\circ} \mathrm{C} / \mathrm{h}$ abolished these beneficial effects. The present findings included significantly attenuated short- and long-term brain injury, and improved NDS and cognitive function compared with the control group, which is consistent with previous studies $(9-11,22)$. There is little doubt that hypothermia improves the outcomes of CPR. It is also hypothesized that if the optimal strategies of cooling and rewarming rate were used for this present study, an increased benefit of EIso combined with TH may have been achieved.

Patients receive $\mathrm{TH}$ treatment in hospital following ROSC, and fentanyl and midazolam are used for prevention of pain, vasoconstriction and shivering (14). However, mild hypothermia alters the pharmacokinetics of these drugs and reduces the systemic clearance of fentanyl and midazolam in rats subsequent to $\mathrm{CA}$ (36). Therefore, for CA patients who are undergoing $\mathrm{TH}$, midazolam may be administered as an overdose, which is accompanied with delaying in awakening, prolonging mechanical ventilation and other subsequent complications (14). Analgesic and sedative drugs combined with TH should therefore have short half-life, which is more beneficial and controllable. (14) EIso was one such agent. A previous study has revealed that EIso treatment enabled more easily controlled depth of anesthesia than isoflurane inhalation for rapid anesthetic induction, and recovery of anesthesia following intravenous EIso administration was faster than following treatment with propofol (37). From the perspective of of TH sedation, EIso may achieve improved results during $\mathrm{TH}$ in hospital (in the intensive care unit or elsewhere) subsequent to $\mathrm{CA}$. Additional studies are required evaluate the clinical effects of combined therapy.

It is believed that the current findings are notable for three reasons. First, the present study demonstrates the synergistic effects of these two interventions in a clinically relevant animal model of cerebral ischemia/reperfusion injury using CA and subsequent CPR. Secondly, for inhaled anesthetics, the need for a vaporizer and associated equipment may limit its use in out-of-hospital CA resuscitation. Furthermore, by circumventing the anesthesia circuitry and the lung's functional residual capacity, intravenous administration of volatile anesthetics may accelerate the induction of anesthesia (38). Intravenous EIso, may offset these limitations and provide rapid anesthetic induction and recovery. Finally, beyond its neuroprotective effects, EIso offers multiple-organ protection, especially in the hemorrhagic shock model. For the synergistic effects of these two interventions, EIso makes TH more applicable. 
There are several limitations of the present study. Firstly, there are different metabolic and rheological properties between the small rodent brain and the complex human brain. Secondly, the results were achieved in healthy animals, which preclude a direct translation to humans, many of whom have comorbidities. Findings analogous to those from the rat CA model are yet to be reported in larger animal and clinical studies.

In conclusion, the present results demonstrated that in a clinically relevant, rat model of CA and CPR, a combination of EIso and $\mathrm{TH}$ had an additive effect in improving both survival and neurological outcomes following ROSC compared with EIso or $\mathrm{TH}$ alone. In contrast to inhaled anesthetics, EIso provided more benefits to utilize the advantages of TH therapy for CA patients.

\section{Acknowledgements}

The present study was supported by a research grant from the National Natural Science Foundation of China (no. 81101403).

\section{References}

1. Fishman GI, Chugh SS, Dimarco JP, Albert CM, Anderson ME, Bonow RO, Buxton AE, Chen PS, Estes M, Jouven X, et al: Sudden cardiac death prediction and prevention: Report from a national heart, lung and blood institute and Heart Rhythm Society Workshop. Circulation 122: 2335-2348, 2010.

2. Xu K and LaManna JC: The loss of hypoxic ventilatory responses following resuscitation after cardiac arrest in rats is associated with failure of long-term survival. Brain Res 1258: 59-64, 2009.

3. Bernard SA, Gray TW, Buist MD, Jones BM, Silvester W, Gutteridge G and Smith K: Treatment of comatose survivors of out-of-hospital cardiac arrest with induced hypothermia. N Engl J Med 346: 557-563, 2002.

4. Hypothermia after Cardiac Arrest Study Group: Mild therapeutic hypothermia to improve the neurologic outcome after cardiac arrest. N Engl J Med 346: 549-556, 2002.

5. Fang AY, Gonzalez FF, Sheldon RA and Ferriero DM: Effects of combination therapy using hypothermia and erythropoietin in a rat model of neonatal hypoxia-ischemia. Pediatr Res 73: 12-17, 2013.

6. Merchant RM, Soar J, Skrifvars MB, Silfvast T, Edelson DP, Ahmad F, Huang KN, Khan M, Vanden Hoek TL, Becker LB and Abella BS: Therapeutic hypothermia utilization among physicians after resuscitation from cardiac arrest. Crit Care Med 34: 1935-1940, 2006.

7. Batista LM, Lima FO, Januzzi JL Jr, Donahue V, Snydeman C and Greer DM: Feasibility and safety of combined percutaneous coronary intervention and therapeutic hypothermia following cardiac arrest. Resuscitation 81: 398-403, 2010.

8. Wolfrum S, Pierau C, Radke PW, Schunkert H and Kurowski V: Mild therapeutic hypothermia in patients after out-of-hospital cardiac arrest due to acute ST-segment elevation myocardial infarction undergoing immediate percutaneous coronary intervention. Crit Care Med 36: 1780-1786, 2008.

9. Tsai MS, Huang CH, Tsai CY, Chen HW, Cheng HJ, Hsu CY, Chang WT and Chen WJ: Combination of intravenous ascorbic acid administration and hypothermia after resuscitation improves myocardial function and survival in a ventricular fibrillation cardiac arrest model in the rat. Acad Emerg Med 21: 257-265, 2014

10. Hayashida K, Sano M, Kamimura N, Yokota T, Suzuki M, Ohta S, Fukuda K and Hori S: Hydrogen inhalation during normoxic resuscitation improves neurological outcome in a rat model of cardiac arrest independently of targeted temperature management. Circulation 130: 2173-2180, 2014.

11. Hayashida K, Sano M, Kamimura N, Yokota T, Suzuki M, Maekawa Y, Kawamura A, Abe T, Ohta S, Fukuda K and Hori S: $\mathrm{H}(2)$ gas improves functional outcome after cardiac arrest to an extent comparable to therapeutic hypothermia in a rat model. J Am Heart Assoc 1: e003459, 2012

12. Fries M, Brücken A, Çizen A, Westerkamp M, Löwer C, Deike-Glindemann J, Schnorrenberger NK, Rex S, Coburn M, Nolte KW, et al: Combining xenon and mild therapeutic hypothermia preserves neurological function after prolonged cardiac arrest in pigs. Crit Care Med 40: 1297-1303, 2012.
13. Meybohm P, Gruenewald M, Albrecht M, Zacharowski KD, Lucius R, Zitta K, Koch A, Tran N, Scholz J and Bein B: Hypothermia and postconditioning after cardiopulmonary resuscitation reduce cardiac dysfunction by modulating inflammation, apoptosis and remodeling. PLoS One 4: e7588, 2009.

14. Zhang YJ, Wu MJ, Li Y and Yu H: Cardiocerebral protection by emulsified isoflurane during cardiopulmonary resuscitation. Med Hypotheses 84: 20-24, 2015.

15. Huang H, Li R, Liu J, Zhang W, Liao T and Yi X: A phase I, dose-escalation trial evaluating the safety and efficacy of emulsified isoflurane in healthy human volunteers. Anesthesiology 120: 614-625, 2014.

16. Chiari PC, Pagel PS, Tanaka K, Krolikowski JG, Ludwig LM, Trillo RA Jr, Puri N, Kersten JR and Warltier DC: Intravenous emulsified halogenated anesthetics produce acute and delayed preconditioning against myocardial infarction in rabbits. Anesthesiology 101: 1160-1166, 2004.

17. Rao Y, Wang YL, Zhang WS and Liu J: Emulsified isoflurane produces cardiac protection after ischemia-reperfusion injury in rabbits. Anesth Analg 106: 1353-1359, 2008.

18. Hu ZY, Luo NF and Liu J: The protective effects of emulsifled isoflurane on myocardial ischemia and reperfusion injury in rats. Can J Anaesth 56: 115-125, 2009.

19. Liu X, Guo QL, Zhang Z, Long L and Yang Y: Effect of emulsified isoflurane on apoptosis of anoxia-reoxygenation neonatal rat cardiomyocytes. Asian Pac J Trop Med 6: 977-981, 2013.

20. Qin Z, Lv E, Zhan L, Xing X, Jiang J and Zhang M: Intravenous pretreatment with emulsified isoflurane preconditioning protects kidneys against ischemia/reperfusion injury in rats. BMC Anesthesiol 14: 28, 2014.

21. Zhang L, Luo N, Liu J, Duan Z, Du G, Cheng J, Lin H and Li Z: Emulsified isoflurane preconditioning protects against liver and lung injury in rat model of hemorrhagic shock. J Surg Res 171: 783-790, 2011.

22. Lu X, Ma L, Sun S, Xu J, Zhu C and Tang W: The effects of the rate of postresuscitation rewarming following hypothermia on outcomes of cardiopulmonary resuscitation in a rat model. Crit Care Med 42: e106-e113, 2014.

23. Che D, Li L, Kopil CM, Liu Z, Guo W and Neumar RW: Impact of therapeutic hypothermia onset, and duration on survival, neurologic function and neurodegeneration after cardiac arrest. Crit Care Med 39: 1423-1430, 2011.

24. Kandel L, Chortkoff BS, Sonner J, Laster MJ and Eger EI II: Nonanesthetics can suppress learning. Anesth Analg 82: 321-326, 1996.

25. Pan H, Chen D, Liu B, Xie X, Zhang J and Yang G: Effects of sodium hydrosulfide on intestinal mucosal injury in a rat model of cardiac arrest and cardiopulmonary resuscitation. Life Sci 93: 24-29, 2013.

26. Zhou C, Huang H, Liu J, Wang $X$, Chen $X$ and Zhang W: Emulsified isoflurance increases convulsive thresholds of lidocaine and produces neural protection after convulsion in rats. Anesth Analg 118: 310-317, 2014.

27. Oddo M, Ribordy V, Feihl F, Rossetti AO, Schaller MD, Chioléro R and Liaudet L: Early predictors of outcome in comatose survivors of ventricular fibrillation and non-ventricular fibrillation cardiac arrest treated with hypothermia: A prospective study. Crit Care Med 36: 2296-2301, 2008

28. Peberdy MA, Callaway CW, Neumar RW, Geocadin RG, Zimmerman JL, Donnino M, Gabrielli A, Silvers SM, Zaritsky AL, Merchant R, et al: Part 9: Post-cardiac arrest care: 2010 American Heart Association Guidelines for cardiopulmonary resuscitation and emergency cardiovascular care. Circulation 122 (18 Suppl 3): S768-S786, 2010.

29. Arrich J, Holzer M, Havel C, Müllner M and Herkner H: Hypothermia for neuroprotection in adults after cardiopulmonary resuscitation. Cochrane Database Syst Rev 2: CD004128, 2016.

30. Nielsen N, Friberg H, Gluud C, Herlitz J and Wetterslev J: Hypothermia after cardiac arrest should be further evaluated-a systematic review of randomised trials with meta-analysis and trial sequential analysis. Int J Cardiol 151: 333-341, 2011.

31. Ma L, Lu X, Xu J, Sun S and Tang W: Improved cardiac and neurological outcomes with postresuscitation infusion of cannabinoid receptor agonist WIN55, 212-2 depend on hypothermia in a rat model of cardiac arrest. Crit Care Med 42: e42-e48, 2014.

32. Hu ZY and Liu J: Effects of emulsified isoflurane on haemodynamics and cardiomyocyte apoptosis in rats with myocardial ischaemia. Clin Exp Pharmacol Physiol 36: 776-783, 2009. 
33. Lu J, Shen Y, Qian HY, Liu LJ, Zhou BC, Xiao Y, Mao JN, An GY, Rui MZ, Wang T and Zhu CL: Effects of mild hypothermia on the ROS and expression of caspase-3 mRNA and LC3 of hippocampus nerve cells in rats after cardiopulmonary resuscitation. World J Emerg Med 5: 298-305, 2014.

34. Soleimanpour H, Rahmani F, Safari S and Golzari SE: Hypothermia after cardiac arrest as a novel approach to increase survival in cardiopulmonary cerebral resuscitation: A review. Iran Red Crescent Med J 16: e17497, 2014.

35. Idris AH, Becker LB, Ornato JP, Hedges JR, Bircher NG, Chandra NC, Cummins RO, Dick W, Ebmeyer U, Halperin HR, et al: Utstein-style guidelines for uniform reporting of laboratory CPR research. A statement for healthcare professionals from a task force of the American Heart Association, the American College of Emergency Physicians, the American College of Cardiology, the European Resuscitation Council, the Heart and Stroke Foundation of Canada, the Institute of Critical Care Medicine, the Safar Center for Resuscitation Research and the Society for Academic Emergency Medicine. Circulation 94: 2324-2336, 1996.
36. Empey PE, Miller TM, Philbrick AH, Melick JA, Kochanek PM and Poloyac SM: Mild hypothermia decreases fentanyl and midazolam steady-state clearance in a rat model of cardiac arrest. Crit Care Med 40: 1221-1228, 2012.

37. Zhou JX, Luo NF, Liang XM and Liu J: The efficacy and safety of intravenous emulsified isoflurane in rats. Anesth Analg 102: 129-134, 2006.

38. Hu ZY, Abbott GW, Fang YD, Huang YS and Liu J: Emulsified isoflurane postconditioning produces cardioprotection against myocardial ischemia-reperfusion injury in rats. J Physiol Sci 63: 251-261, 2013. 